\title{
LAS AGRUPACIONES URBANAS ESPAÑOLAS Y LA ESTRUCTURA DE SU APARATO DE DISTRIBUCION COMERCIAL
}

\author{
$381: 311(46)$
}

por

\section{Ignacio Ballester Ros}

SUMARIO: I. INTRODUCCION.-II. LAS AGRUPACIONES URBANAS ESPAÑOLAS: 1. CONSIDERACIONES GENERALES. 2. SU CONFIGURACIÓN. 3. Su eVOLUCí́n demográfica. 4. Otras caracterfSTicas.-III. ESTRUCTURA DEL APARATO DE DISTRIBUCION COMERCIAL: 1. ESTABLECIMIENTOS EN RÉGIMEN DE AUTOSERVICIO. 2. ESTABLECIMIENTOS DE GRAN SUPERFICIE.-IV. ESTIMACION DEL CONSUMO PRIVADO.

\section{INTRODUCCION}

Desde su aparición en el año 1965, dedicamos todos los años un comentario al Anuario del Mercado Español, que al servicio de la política comercial de las empresas, se edita bajo el patrocinio de Banesto; y nos detenemos, de manera especial, en el análisis de los resultados publicados con referencia a algunas de las investigaciones especiales realizadas por el equipo de economistas y estadísticos que lo elaboran.

En la nueva edición, que acaba de aparecer, correspondiente al presente año, se mantiene la estructura tradicional de la obra con estudios, de carácter permanente, relativos a la cuota de mercado, consumos energéticos, licencias comerciales, índices de capacidad 
de compra y de riqueza activa, indicadores de mercado, análisis de la coyuntura, con determinación del índice de vivacidad y de los movimientos migratorios interiores, todos ellos con especial referencia a las áreas comerciales españolas.

Contiene, además, tres trabajos de indudable interés local. El primero consiste en la información estadística sobre los Municipios españoles con población superior a 3.000 habitantes, que se elabora con periodicidad bienal. El segundo es un estudio, que se repite cada cinco años, sobre los niveles de renta per capita de los Municipios españoles, agrupados por áreas comerciales y $\mathrm{Re}$ giones mercadológicas, que constituye «la aportación más pormenorizada que acerca de la distribución espacial de la renta existe en nuestro país".

El último trabajo, que constituye una aportación nueva, tiende a un mejor conocimiento de la estructura mercadológica española, y consiste en la delimitación de las agrupaciones urbanas más importantes, el análisis de su aparato comercial y la estimación de sus respectivos consumos privados. Por el extraordinario interés de los tres aspectos que contempla, dedicamos a él el comentario de esta crónica estadística.

\section{LAS AGRUPACIONES URBANAS ESPAÑOLAS}

\section{CoNSIDERACIONES GENERALES}

Al-presentar los resultados de esta investigación se hace referencia, en el propio Anuario, al proceso de urbanización, iniciado en todo el mundo, a principio del siglo XIX, merced al desarrollo industrial, que da lugar, mediante el "transferimiento de población rural, al nacimiento de grandes núcleos urbanos, que ofrecen mayores posibilidades de trabajo estable y mejor remuneradon; se alude a «la evolución de la población mundial, duplicada entre 1800 y 1950, que se sitúa en este último año en 2.500 millones de habitantes, alcanza los 4.000 en 1977 y se prevé supere los 6.000 a principios del próximo siglo»; y se subraya, en consecuencia, «el espectacular desarrollo del «índice de urbanización», con la existencia en 1970 de más de 70 aglomeraciones urbanas más que millonarias» y la ubicación «de más del 70 por 100 de la población mundial en ciudades de más de 100.000 habitantes». 
Igualmente se destaca cómo «el crecimiento indefinido del espacio urbano ha generado, junto a altas tasas de densidad", la formación de "puntos de condensación" y el desarrollo del fenómeno de la "conurbación», al desbordar las grandes ciudades sus límites administrativos e invadir los términos municipales colindantes, soldando núcleos urbanos tradicionalmente separados por zonas despobladas y dando lugar a las «aglomeraciones urbanas», constituidas por «un núcleo base y el entorno de Municipios circundantes que, aunque conservan su autonomía administrativa, presentan una estructura y un comportamiento general uniformes, contribuyendo a forjar su unidad la facilidad de transportes, la disponibilidad de servicios comunes y las conexiones de orden laboral, social y económico».

En estas aglomeraciones, su periferia se halla en contacto con el habitat rural; pero nada tiene que ver con el trabajo de la tierra. En ella se sitúan tanto las "ciudades dormitorio» como las zonas residenciales urbanizadas, ambas huyendo del centro de las grandes urbes.

Por lo que a España se refiere, el fenómeno de creciente urbanización le ha afectado, especialmente a partir de 1960, debido a la intensificación de los movimientos migratorios interiores, que se iniciaron en la segunda mitad del siglo xx. A partir de la citada fecha se han transferido a núcleos urbanos, con la agravante de fuertes desequilibrios zonales, más de seis millones de habitantes. Entre 1900 y 1970, la distribución de la población española ha registrado cambios trascendentales, ya que la población rural pasó del 27,5 al 11,0 por 100 del total, quedando reducida a la mitad, mientras que la urbana creció del 32,2 al 66,5 por 100 , duplicándose. En 1975 la población residente en núcleos de más de 10.000 habitantes supone el 65 por 100 de la población rural. En el conjunto de cinco Municipios: Madrid, Barcelona, Bilbao, Valencia y Sevilla se ubica el 21 por 100 de la población.

En todo caso, el proceso de urbanización afecta directamente a la política de equipamiento colectivo, y muy especialmente al ámbito mercadológico y a la conformación espacial del comercio. 


\section{SU CONFIGURACIÓN}

No es objeto de este trabajo definir las aglomeraciones urbanas existentes en España. Sólo se ha pretendido, a título de ensayo, describir las «agrupaciones urbanas, constituidas por aquellos Municipios contiguos que, por las características de la mayoría de sus habitantes, presentan aspectos urbanos que, en conjunto, constituyen, en cierto modo, una unidad».

El estudio se inició, en un primer momento, «con el análisis de todos los Municipios de población igual o superior a 50.000 habitantes, por entender que es a partir de este nivel poblacional donde se comienza a entrever este fenómeno de la agrupación humana de Municipios colindantes». Con respecto a este nivel de Municipios se tomaron en consideración los siguientes datos: población y densidad de población, viviendas en edificaciones de cinco o más viviendas y en edificaciones de utilización agraria, licencias comerciales, teléfonos instalados y peticiones pendientes, distancia de cada Municipio al centro de la urbe principal, nivel medio de renta per capita y cuota de mercado, medios de comunicación y transporte e intensidad de tráfico, intercambio diario de personas entre los Municipios colindantes, localización de las industrias y personal empleado en ellas y grado de desarrollo de los servicios públicos.

En la etapa final, se estimó que se justifica la agrupación de Municipios cuando en ellos se da la mayor parte de las siguientes circunstancias: predominio de estructura urbana en las construcciones, uso común de varios servicios públicos, escasa ruralidad, facilidad de traslado diario, proximidad y existencia de relaciones frecuentes entre ellos, nivel de desarrollo análogo, crecimiento demográfico rápido y existencia de un aparato comercial complementario. $\mathrm{Y}$ se tomó como cifra mínima de población para que un solo Municipio principal y los de su entorno sea considerada como «agrupación urbana» la de 150.000 habitantes, «por estimar que a partir de esta cifra comienza a existir la fuerza de cohesión suficiente para considerar que existe una unidad de hecho, que es la premisa que justifica la formación de estas Agrupaciones».

Las Agrupaciones urbanas configuradas y los Municipios que comprende cada una de ellas son: 
La Coruña.-La Coruña, Arteijo, Culleredo, Oleiros.

Vigo.-Vigo.

Gijón.-Gijón.

Oviedo.-Oviedo, Llanera, Noreña, Ribera de Arriba.

Santander.-Santander, Astillero, Camargo, Marina de Cudeyo, Santa Cruz de Bezana.

Bilbao.-Bilbào, Arrigorriaga, Baracaldo, Basauri, Berango, Echévarri, Galdácano, Guecho, Lejona, Miravalles, Plencia, Portugalete, San Salvador del Valle, Santurce-Antiguo, Santurce-Ortuella, Sestao, Sopelana, Urdúliz, Zarátamo.

Vitoria.-Vitoria, Arrazua-Ubarrundía, Foronda.

San Sebastián.-San Sebastián, Lezo, Pasajes, Rentería, Usúrbil.

Pamplona.-Pamplona, Ansoaín, Burlada, Elorz, Villava.

Zaragoza.-Zaragoza, Cuarte de Huerva.

Sabadell-Tarrasa.-Sabadell, Tarrasa, Castellar del Vallés, Matadepera, San Quirico de Tarrasa, Santa María de Barbará, Santa Perpetua de Moguda.

Barcelona.-Barcelona, Badalona, Cornellá, Esplugas de Llobregat, Hospitalet, Moncada y Reixach, Prat de Llobregat, San Adrián de Besós, San Baudilio de Llobregat, San Justo Desvern, Santa Coloma de Gramanet.

Palma de Mallorca.-Palma de Mallorca, Calviá, Lluchmayor, Marratxi.

Valladolid.-Valladolid, Arroyo, Cistérniga, Laguna de Duero, Renedo, Santovenia de Pisuerga, Zaratán.

Madrid.-Madrid, Alcobendas, Alcorcón, Boadilla del Monte, Coslada, Majadahonda, Móstoles, Pozuelo de Alarcón, Rozas de Madrid (Las), San Fernando de Henares, San Sebastián de los Reyes, Torrejón de Ardoz, Fuenlabrada, Getafe, Leganés.

Castellón.-Castellón, Almazora, Villarreal de los Infantes.

Valencia.-Valencia, Albal, Alboraya, Alfafar, Benetúser, Bonrepós y Mirambell, Burjasot, Catarroja, Cuart de Poblet, Chirivella, Godella, Manises, Masanasa, Mislata, Paiporta, Paterna, Picaña, Rocafort, Sedaví, Tabernes Blanques.

Alicante.-Alicante, Campello, Muchamiel, San Juan,' San Vicente del Raspeig.

Murcia.-Murcia, Alcantarilla.

Granada.-Granada, Armilla, Cájar, Cenes de la Vega, Churria- 
na de la Vega, Huétor-Vega, Jun, Maracena, Ogíjares, Peligros, Pulianas, Zubia.

Málaga.-Málaga, Benalmádena, Rincón de la Victoria.

Córdoba.-Córdoba.

Sevilla.-Sevilla, La Algaba, Bormujos, Camas, Castilleja de Guzmán, Castilleja de la Cuesta, Coria del Río, Dos Hermanas, Gelves, Ginés, Mairena del Aljarafe, Palomares del Río, La Rinconada, Salteras, San Juan de Aznalfarache, Santiponce, Tomares, Umbrete, Valencina de la Concepción.

Jerez de la Frontera.-Jerez de la Frontera.

Cádiz.-Cádiz, San Fernando.

Santa Cruz de Tenerife.-Santa Cruz de Tenerife, La Laguna, El Rosario.

Las Palmas.-Las Palmas.

En resumen, las 27 Agrupaciones abarcan 165 Municipios, cuyos términos municipales ocupan, en conjunto, una superficie de $11.851,7 \mathrm{~km}^{2}$, el 2,3 por 100 del total de España, y tienen una población de 14.558 .756 habitantes, que supone el 40,6 por 100 de la población total, y una densidad media de 1.228 habitantes por kilómetro cuadrado.

El total de las Agrupaciones, enumeradas con arreglo a un criterio geográfico, es éste:

\begin{tabular}{|c|c|c|c|c|}
\hline Agrupaciones & $\begin{array}{c}\text { Num. de } \\
\text { Municipios }\end{array}$ & $\begin{array}{c}\text { Superficie } \\
\left(\mathrm{km}^{2}\right)\end{array}$ & $\begin{array}{l}\text { Poblacionn } \\
\text { en } 1974\end{array}$ & $\begin{array}{c}\text { Densidad } \\
\text { de población }\end{array}$ \\
\hline 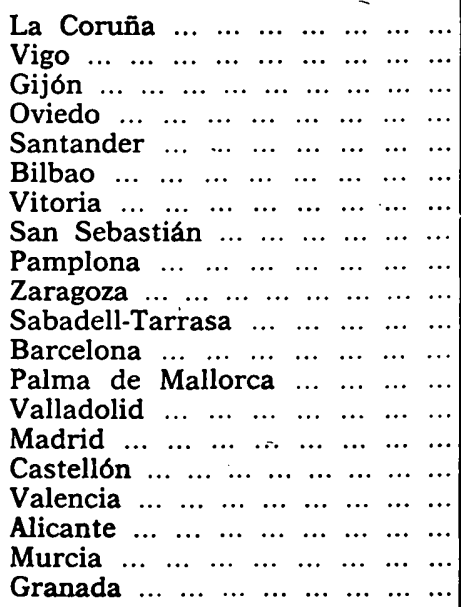 & $\begin{array}{r}4 \\
1 \\
1 \\
4 \\
5 \\
19 \\
3 \\
5 \\
5 \\
2 \\
7 \\
11 \\
4 \\
7 \\
15 \\
3 \\
20 \\
5 \\
2 \\
12\end{array}$ & $\begin{array}{r}237,6 \\
109,4 \\
181,7 \\
307.5 \\
118,5 \\
319,8 \\
295,5 \\
148,9 \\
106,8 \\
1.068,9 \\
215,9 \\
233,4 \\
716,5 \\
332,9 \\
1.208,8 \\
208,4 \\
299,9 \\
352,6 \\
941,7 \\
162,5\end{array}$ & $\begin{array}{r}245.988 \\
219.144 \\
208.061 \\
183.288 \\
205.270 \\
874.662 \\
158.195 \\
238.026 \\
188.855 \\
526.677 \\
369.219 \\
2.688 .365 \\
260.174 \\
276.332 \\
3.725 .388 \\
153.457 \\
934.739 \\
232.419 \\
289.357 \\
239.910\end{array}$ & $\begin{array}{r}1.035 \\
2.003 \\
1.145 \\
596 \\
1.732 \\
2.735 \\
535 \\
1.598 \\
1.768 \\
492 \\
1.710 \\
11.518 \\
363 \\
830 \\
3.082 \\
736 \\
3.116 \\
659 \\
841 \\
1.476\end{array}$ \\
\hline
\end{tabular}




\begin{tabular}{|c|c|c|c|c|}
\hline Agrupaciones & $\begin{array}{c}\text { Núm. de } \\
\text { Municipios }\end{array}$ & $\begin{array}{c}\text { Superficie } \\
\left(\mathrm{km}^{2}\right)\end{array}$ & $\begin{array}{c}\text { Poblacion } \\
\text { en } 1974\end{array}$ & $\begin{array}{c}\text { Densidad } \\
\text { de población }\end{array}$ \\
\hline 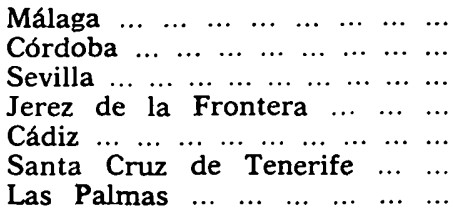 & $\begin{array}{r}3 \\
1 \\
19 \\
1 \\
2 \\
3 \\
1\end{array}$ & $\begin{array}{r}459,6 \\
1.244,6 \\
697,0 \\
1.410,0 \\
40,5 \\
333,9 \\
98,9\end{array}$ & $\begin{array}{l}413.185 \\
248.292 \\
750.388 \\
159.676 \\
206.206 \\
257.676 \\
305.807\end{array}$ & $\begin{array}{r}899 \\
199 \\
1.076 \\
113 \\
5.091 \\
771 \\
3.092\end{array}$ \\
\hline
\end{tabular}

Señalemos que 18 Agrupaciones - las dos terceras partes- son periféricas, y las restantes nueve, interiores. Por Regiones se distribuyen así: siete en la galaico-cántabra; tres en el Valle del Ebro; siete en Cataluña, Valencia, Baleares y Murcia; dos en Castilla; seis en Andalucía, y dos en Canarias.

\section{SU EVOLUCIÓN DEMOGRAFICA}

En la tabla anterior se observa el desigual tamaño y el distinto grado de concentración demográfica de estas Agrupaciones urbanas, y cómo resaltan por su extraordinaria importancia las de Madrid y Barcelona, que, en conjunto, albergan a casi seis millones y medio de habitantes, cerca del 18 por 100 de la población total.

La evolución de la población del conjunto nacional y de las Agrupaciones consideradas ha sido, en los años de referencia, y expresada en miles de habitantes, la siguiente:

\begin{tabular}{|c|c|c|c|c|}
\hline & $\begin{array}{l}\text { Conjunto } \\
\text { nacional }\end{array}$ & Indices & $\begin{array}{l}\text { Agrupa- } \\
\text { ciones }\end{array}$ & Indices \\
\hline $\begin{array}{llllllllll}1960 & \ldots & \ldots & \ldots & \ldots & \ldots & \ldots & \ldots & \ldots & \ldots \\
1970 & \ldots & \ldots & \ldots & \ldots & \ldots & \ldots & \ldots & \ldots & \ldots \\
1974 & \ldots & \ldots & \ldots & \ldots & \ldots & \ldots & \ldots & \ldots & \ldots\end{array}$ & $\begin{array}{l}30.583 \\
33.956 \\
35.833\end{array}$ & $\begin{array}{l}100,0 \\
111,0 \\
117,2\end{array}$ & $\begin{array}{r}9.900 \\
13.527 \\
14.559\end{array}$ & $\begin{array}{l}100,0 \\
136,6 \\
147,1\end{array}$ \\
\hline
\end{tabular}

La población de las Agrupaciones ha seguido una evolución creciente en los tres últimos lustros, aunque con menos intensidad en el último de ellos. En 1960 su población global representaba el 32,4 por 100 del total; en 1970 alcanzaba el 39,8, que se elevaba al 40,6 en 1974.

Es de resaltar el hecho de que mientras la población total aumentó en el período 1960-74 sólo el 17,2 por 100, la de las Agrupaciones se incrementó en el 47,1, lo que denota la mayor fuerza de atracción demográfica de las Agrupaciones.

Se registra, sin embargo, una evidente falta de homogeneidad 
entre las Agrupaciones en orden al grado de incremento que, en el mismo período, ha tenido cada una de ellas, lo que permite clasificarlas de esta forma:

Con aumento menor del 25 por 100: La Coruña, Murcia, Jerez de la Frontera y Cádiz.

Con incremento entre el 25 y el 47 por 100: Oviedo, Santander, San Sebastián, Barcelona, Palma de Mallorca, Valencia, Granadá, Málaga, Córdoba, Sevilla y Santa Cruz de Tenerife.

Con aumento por encima del promedio nacional: Vigo, Gijón, Bilbao, Vitoria, Pamplona, Zaragoza, Sabadell-Tarrasa, Valladolid, Madrid, Castellón, Alicante y Las Palmas.

El incremento máximo corresponde a Vitoria, y el mínimo a Murcia.

En la siguiente tabla se detalla la población del núcleo principal y la de los demás Municipios de cada Agrupación, así como su distribución porcentual:

\begin{tabular}{|c|c|c|c|c|}
\hline \multirow[b]{2}{*}{ Agrupaciones } & \multicolumn{4}{|c|}{ Población en 1974} \\
\hline & $\begin{array}{l}\text { Del núcleo } \\
\text { principal }\end{array}$ & Porcentajes & $\begin{array}{c}\text { En los demas } \\
\text { Municipios }\end{array}$ & Porcentajes \\
\hline 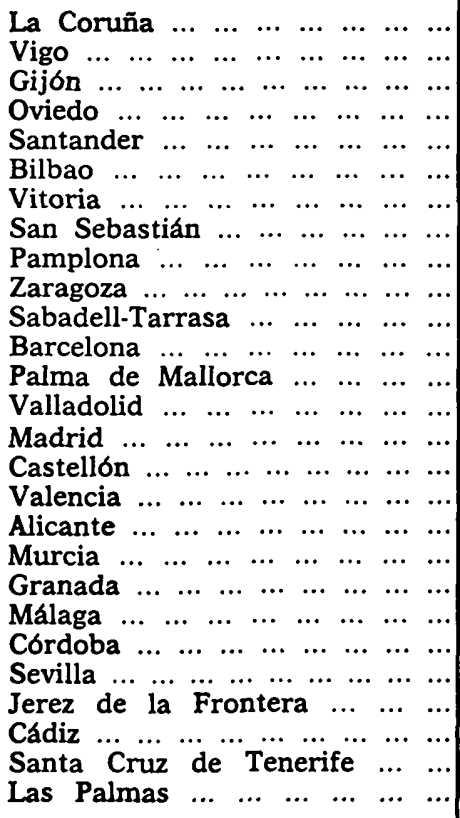 & $\begin{array}{r}211.581 \\
219.144 \\
208.161 \\
166.812 \\
170.814 \\
431.061 \\
156.673 \\
167.529 \\
159.282 \\
625.847 \\
332.942 \\
1.816 .623 \\
235.932 \\
268.428 \\
3.274 .043 \\
103.078 \\
679.829 \\
195.490 \\
267.916 \\
199.837 \\
397.643 \\
248.292 \\
580.301 \\
159.676 \\
145.153 \\
167.283 \\
305.807\end{array}$ & $\begin{array}{r}86,0 \\
100,0 \\
100,0 \\
91,0 \\
83,2 \\
49,3 \\
99,0 \\
70,4 \\
84,3 \\
99,8 \\
90,2 \\
67,6 \\
90,7 \\
97,1 \\
87,9 \\
67,2 \\
72,7 \\
84,1 \\
92,6 \\
83,3 \\
96,2 \\
100,0 \\
77,3 \\
100,0 \\
70,4 \\
64,9 \\
100,0\end{array}$ & $\begin{array}{c}34.407 \\
- \\
16.476 \\
34.396 \\
443.601 \\
1.522 \\
70.497 \\
29.573 \\
830 \\
36.277 \\
871.742 \\
24.242 \\
7.904 \\
451.345 \\
50.379 \\
254.910 \\
36.929 \\
21.441 \\
40.073 \\
15.542 \\
170.087 \\
- \\
61.053 \\
90.393 \\
-\end{array}$ & $\begin{array}{r}14,0 \\
- \\
\overline{9,0} \\
16,8 \\
50,7 \\
1,0 \\
29,6 \\
15,7 \\
0,2 \\
9,8 \\
32,4 \\
9,3 \\
2,9 \\
12,1 \\
32,8 \\
27,3 \\
15,9 \\
7,4 \\
16,7 \\
3,8 \\
\overline{22,7} \\
\overline{29,6} \\
35,1 \\
-\end{array}$ \\
\hline & 11.795 .137 & 81,0 & 2.763.619 & 19,0 \\
\hline
\end{tabular}


Del examen de las cifras anteriores se deduce que el núcleo principal comprende más de las cuatro quintas partes de la población de las Agrupaciones, lo que afecta a 19 de ellas.

Eliminadas las cinco Agrupaciones constituidas por un solo Municipio, se sitúan por encima del promedio nacional 14 Agrupaciones, mientras que otras ocho reflejan una menor importancia demográfica del núcleo principal $\mathrm{y}$, en consecuencia, un mayor peso de la población de los Municipios colindantes y periféricos, que oscila entre la quinta y la tercera parte, aunque en el caso de Bilbao supera a la mitad de la población de la Agrupación. Parece advertirse, tal vez, una tendencia al desplazamiento de la población desde el núcleo principal hacia la periferia en algunas Agrupaciones.

En la tabla siguiente se recogen, en primer lugar, los porcentajes de incremento anual de la población de las Agrupaciones en el decenio 1960-70 y en el quinquenio 1970-74. Los promedios nacionales indican claramente que el aumento medio anual fue muy importante en el citado decenio; pero que en el quinquenio siguien-

\begin{tabular}{|c|c|c|c|c|}
\hline \multirow{3}{*}{ Agrupaciones } & \multicolumn{4}{|c|}{ PORCENTAJES } \\
\hline & \multicolumn{2}{|c|}{$\begin{array}{l}\text { De incremento medio } \\
\text { anual de la población }\end{array}$} & \multicolumn{2}{|c|}{$\begin{array}{c}\text { De viviendas sobre } \\
\text { el total }\end{array}$} \\
\hline & $1960-70$ & $1970-74$ & $\begin{array}{l}\text { En edifi- } \\
\text { cios de } \\
50 \text { mds } \\
\text { viviendas }\end{array}$ & $\begin{array}{l}\text { En edifi- } \\
\text { cios de } \\
\text { utilización } \\
\text { agraria }\end{array}$ \\
\hline 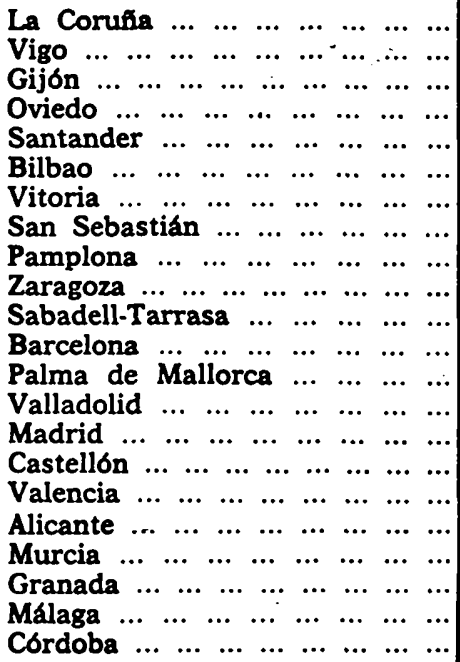 & $\begin{array}{l}0,7 \\
3,6 \\
5,0 \\
2,0 \\
2,5 \\
4,6 \\
6,3 \\
3,2 \\
6,8 \\
4,7 \\
5,1 \\
3,1 \\
4.6 \\
5,3 \\
4,8 \\
4,4 \\
3,6 \\
5,2 \\
1,8 \\
2,3 \\
2,6 \\
1,9\end{array}$ & $\begin{array}{l}2,7 \\
2,8 \\
2,7 \\
1,9 \\
3,1 \\
2,1 \\
3,2 \\
0,8 \\
2,0 \\
2,4 \\
3,8 \\
1,8 \\
0,1 \\
3,3 \\
1,9 \\
2,4 \\
1,6 \\
1,5 \\
2,6 \\
1,2 \\
1,5 \\
1,4\end{array}$ & $\begin{array}{l}62,5 \\
38,2 \\
77,6 \\
64,3 \\
74,9 \\
83,3 \\
85,7 \\
88,5 \\
87,1 \\
82,4 \\
40,3 \\
40,3 \\
50,4 \\
82,8 \\
87,1 \\
40,4 \\
75,9 \\
70,5 \\
28,5 \\
59,4 \\
67,9 \\
61,1\end{array}$ & $\begin{array}{r}3,8 \\
6,6 \\
2,5 \\
5,0 \\
4,0 \\
1,5 \\
2,3 \\
1,9 \\
0,9 \\
1,7 \\
0,5 \\
0,1 \\
1,9 \\
0,7 \\
0,1 \\
2,5 \\
2,3 \\
0,4 \\
15,0 \\
5,7 \\
10,6 \\
2,8\end{array}$ \\
\hline
\end{tabular}




\begin{tabular}{|c|c|c|c|c|}
\hline \multirow{3}{*}{ Agrupaciones } & \multicolumn{4}{|c|}{ PORCENTAJES } \\
\hline & \multicolumn{2}{|c|}{$\begin{array}{l}\text { De incremento medio } \\
\text { anual de la población }\end{array}$} & \multicolumn{2}{|c|}{$\begin{array}{c}\text { De viviendas sobre } \\
\text { el total }\end{array}$} \\
\hline & $1960-70$ & $1970-74$ & $\begin{array}{l}\text { En edifi- } \\
\text { cios de } \\
50 \text { más } \\
\text { viviendas }\end{array}$ & $\begin{array}{l}\text { En edifi- } \\
\text { cios de } \\
\text { utilización } \\
\text { agraria }\end{array}$ \\
\hline $\begin{array}{llllllllll}\text { Sevilla } & \ldots & \ldots & \ldots & \ldots & \ldots & \ldots & \ldots & \ldots & \ldots \\
\text { Jerez } & \text { de } & \text { la } & \text { Frontera } & \ldots & \ldots & \ldots \\
\text { Cádiz } & \ldots & \ldots & \ldots & \ldots & \ldots & \ldots & \ldots & \ldots & \ldots \\
\text { Santa } & \text { Cruz } & \text { de } & \text { Tenerife } & \ldots & \ldots \\
\text { Las } & \text { Palmas } & \ldots & \ldots & \ldots & \ldots & \ldots & \ldots \\
& & & & & & & \\
& & \text { Media } & \ldots & \ldots & \ldots & \ldots & \ldots & \ldots\end{array}$ & $\begin{array}{l}2,6 \\
1,5 \\
1,5 \\
3,2 \\
4,8 \\
3,7\end{array}$ & $\begin{array}{l}1,4 \\
1,6 \\
1,3 \\
1,1 \\
1,6 \\
1,9\end{array}$ & $\begin{array}{l}61,6 \\
53,8 \\
76,0 \\
36,5 \\
53,8\end{array}$ & $\begin{array}{l}0,9 \\
5,3 \\
0,2 \\
0,5 \\
0,2\end{array}$ \\
\hline
\end{tabular}

te el ritmo de crecimiento anual se aminoró mucho, reduciéndose prácticamente a la mitad.

Las Agrupaciones con un incremento medio anual superior al promedio nacional en el período 1960-70 coinciden, en líneas generales, con las enumeradas al tratar de los incrementos globales del período 1960-74 en los primeros párrafos de este apartado. En gran parte se mantienen con altos incrementos, dentro de la tónica apuntada, en el periodo siguiente, si bien a ellas hay que añadir las Agrupaciones de Galicia, Asturias y Cantabria, así como Murcia.

El porcentaje de viviendas en édificios de cinco o más viviendas puede ser un indicativo de mayor grado de urbanización. La distribución de-las : Agrupaciones por esta circunstancia resulta muy dispar. Los valores máximos corresponden a Madrid y Valladolid, en el centro del país, y a las Vascongadas, Navarra y Zaragoza; mientras los valores mínimos corresponden a tres Agrupaciones muy distanciadas geográficamente: Murcia, Vigo y Santa Cruz de Tenerife.

El grado de disponibilidad de edificios con utilización agraria puede expresar un cierto nivel de ruralidad. En este orden sobresalen Murcia y Málaga, y en segundo grado, Vigo, Oviedo y Jerez de la Frontera. 


\section{OTRAS CARACTERISTICAS}

La evolución del número de licencias comerciales ha sido, en miles, la siguiente en los años de referencia:

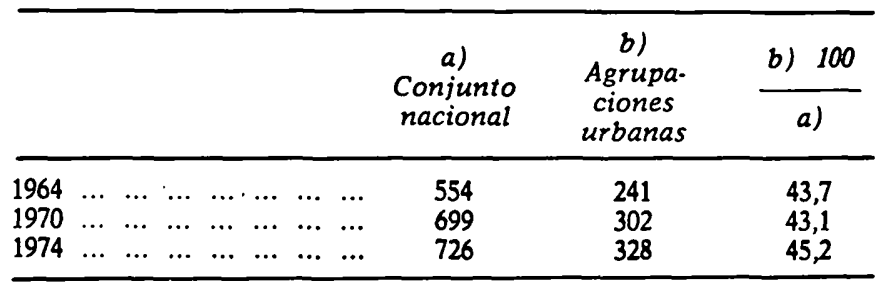

Debe tenerse en cuenta al analizar estas cifras que cada licencia es una autorización para ejercer la actividad comercial sobre bienes y servicios, pero no prejuzga la importancia de los establecimientos en que se ejerce, aunque puede suponerse que los establecimientos más importantes se hallan en las Agrupaciones urbanas consideradas.

Se aprecia un bache en la apertura de establecimientos en 1970, que se corrige en 1974.

El porcentaje de incremento medio anual, que para el conjunto nacional bajó del 4,4 en 1964-70 al 0,9 en 1970-74, disminuyó en los mismos períodos del 4,1 al 2,2 con respecto a las Agrupaciones, 'lo que indica que en el segundo período considerado las Agrupaciones, aun disminuyendo el ritmo de crecimiento, se mantuvieron en una posición más favorable.

En ambos períodos destacan por sus porcentajes de incremento superiores al nacional: Oviedo, Vitoria, Sabadell-Tarrasa, Palma de Mallorca, Alicante y Jerez de la Frontera. Con porcentajes superiores en el primer período aparecen: La Coruña, Gijón, San: tander, Bilbao, Zaragoza, Valladolid, Castellón, Valencia, Granadä, Sevilla, Cádiz, Santa Cruz de Tenerife y Las Palmas. En el segundo período destacan: Vigo, Barcelona, Madrid, Murcia, Granada, Málaga y Córdoba.

Por sus tasas más altas destacan: Gijón, Vitoria, Valladolid y Santa Cruz de Tenerife.

Registran valores negativos en el segundo período: San Sebastián, Pamplona, Valladolid y Santa Cruz de Tenerife.

El promedio nacional de licencias comerciales por 100 habitan- 
tes es de 2,4. Lo igualan o superan las Agrupaciones de Gijón, Oviedo, Bilbao, Zaragoza, Barcelona, Palma de Mallorca, Alicante y Las Palmas.

La distribución de las licencias comerciales por ramas en las Agrupaciones es bastante similar a la del conjunto nacional, si bien debe señalarse la menor proporción de las licencias de alimentación, que se compensa con un mayor número de establecimientos especializados en las primeras.

La evolución del número de teléfonos y de las solicitudes pendientes de instalación ha sido en los años de referencia, ésta, en miles:

\begin{tabular}{|c|c|c|c|}
\hline & \multirow{2}{*}{$\begin{array}{l}\text { a) } \\
\text { Conjunto } \\
\text { nacional }\end{array}$} & \multirow{2}{*}{$\begin{array}{c}\text { b) } \\
\text { Agrupa- } \\
\text { ciones } \\
\text { urbanas }\end{array}$} & \multirow{2}{*}{ b) 100} \\
\hline & & & \\
\hline $\begin{array}{llllllll}1963 & \ldots & \ldots & \ldots & \ldots & \ldots & \ldots & \ldots \\
1969 & \ldots & \ldots & \ldots & \ldots & \ldots & \ldots & \ldots \\
1975 & \ldots & \ldots & \ldots & \ldots & \ldots & \ldots & \ldots\end{array}$ & $\begin{array}{l}2.628 \\
4.713 \\
8.376\end{array}$ & $\begin{array}{l}1.923 \\
3.431 \\
5.508\end{array}$ & $\begin{array}{l}73,2 \\
72,8 \\
65,8\end{array}$ \\
\hline
\end{tabular}

Las Agrupaciones urbanas consideradas disponen de los dos tercios de los teléfonos en 1975, si bien, como se deduce de las cifras anteriores, su peso relativo ha venido reduciéndose desde 1963. El porcentaje de incremento medio anual, que para el conjunto nacional pasó del 13,2 en el período 1963-69 al 19,4 en 1969-75, disminuyó en los mismos períodos del 13,1 al 10,1 con respecto a las Agrupaciones. Ello quiere decir que las zonas rurales y los demás núcleos urbanos han visto incrementadas más rápidamente sus instalaciones telefónicas que los grandes núcleos, lo que parece lógico.

Con porcentajes de incremento medio anual superiores al nacional en ambos períodos, figuran las Agrupaciones de La Coruña, Vigo, Vitoria y Zaragoza, Pamplona, Sabadell-Tarrasa, Alicante y Murcia, Málaga y Las Palmas, que, con alguna excepción, ostentan bajas cuotas de mercado. Con porcentajes superiores solamente en el primer período se sitúan, además, San Sebastián, Barcelona y Palma de Mallorca. Las Agrupaciones con incrementos medios superiores al nacional en el segundo período es mayor, y alcanza a Gijón, Oviedo, Santander, Bilbao, Valladolid, Madrid, Castellón, Valencia, Granada, Córdoba, Sevilla, Jerez de la Frontera, Cádiz y Santa Cruz de Tenerife. Las más altas tasas corresponden en el 
primer período a Vitoria, Palma de Mallorca, Alicante y Las Palms, y en el segundo, a Málaga y Sevilla.

El promedio nacional de teléfonos por 100 habitantes en 1975 es de 37,8. Tan sólo lo superan las Agrupaciones de San Sebastián, Barcelona, Palma de Mallorca, Valladolid y Madrid. Destaca Valladolid por sus elevadas tasas:

La cuota de mercado de las 27 Agrupaciones urbanas es de 52.343, más de la mitad del conjunto nacional. Madrid y Barcelona absorben por sí solas la cuarta parte. Los restantes valores difieren acusadamente entre sí, destacando, a segundo nivel, Bilbao, Valencia y Sevilla; y en un tercer nivel, Zaragoza, Palma de Mallorca, Málaga y Las Palmas.

\section{ESTRUCTURA DEL APARATO DE DISTRIBUCION COMERCIAL}

En esta materia, aparte de un análisis ampliatorio de las licencias comerciales, por ramas, se estudia la estructura y evolución de dos clases de establecimientos: los en régimen de autoservicio, para la alimentación, y los de gran superficie.

Se entiende por autoservicio «la técnica de venta por la que el cliente queda situado directamente y sin intermediarios en presencia del producto, sin ninguna influencia personal del vendedor y de forma tal que la decisión de compra es esencialmente individual y reservada».

Bajo este régimen pueden considerarse: los «autoservicios» propiamente dichos, con una superficie de venta entre 40 y 120 metros cuadrados; los "superservicios», con superficie entre 120 y 400 , y los «supermercados", con superficie superior a los 400 metros cuadrados.

Los establecimientos de agran superficie» se han desarrollado en función de los cambios operados en los hábitos de compra de las amas de casa, tendentes a adquirir artículos de diversa naturaleza en un mismo establecimiento, y la participación de grandes empresas de distribución en el sector comercial, que han abierto al público establecimientos de grandes superficies; todo ello en estrecha conexión con el proceso de concentración urbana y el desarrollo de la motorización. 
Bajo esta denominación se comprenden: el "almacén popular», el «hipermercado» y el "gran almacén», que se distinguen por su distinta superficie.

\section{ESTABLECIMIENTOS EN RÉGIMEN DE AUTOSERVICIO}

El proceso de implantación de los autoservicios ha sido rápido en España, pasando de 80 establecimientos en 1960 a 8.716 en 1975, de ellos 3.963 en las Agrupaciones urbanas, si bien la cifra de supermercados es aún reducida respecto a Europa.

En 1975 los establecimientos y sus principales características eran éstos:

\begin{tabular}{|c|c|c|c|}
\hline & $\begin{array}{c}\text { Autoser- } \\
\text { vicios }\end{array}$ & $\begin{array}{l}\text { Superser- } \\
\text { vicios }\end{array}$ & $\begin{array}{c}\text { Super- } \\
\text { mercados }\end{array}$ \\
\hline \multicolumn{4}{|l|}{ Conjunto nacional: } \\
\hline 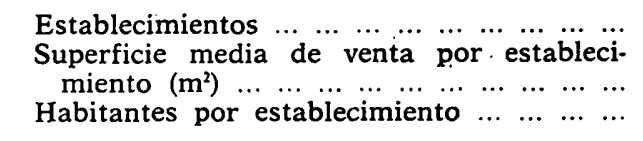 & $\begin{array}{r}6.760 \\
64,8 \\
5.301\end{array}$ & $\begin{array}{l}1.643 \\
180,7 \\
21.810\end{array}$ & $\begin{array}{c}313 \\
707,8 \\
114.483\end{array}$ \\
\hline \multicolumn{4}{|l|}{ Agrupaciones: } \\
\hline 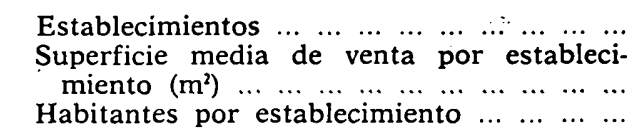 & $\begin{array}{r}3.024 \\
62,6 \\
4.814\end{array}$ & $\begin{array}{c}749 \\
184,4 \\
19.438\end{array}$ & $\begin{array}{r}190 \\
716,4 \\
76.825\end{array}$ \\
\hline
\end{tabular}

Las Agrupaciones disponen del 45 por 100 de autoservicios y superservicios y del 60 por 100 de supermercados; la superficie media de los superservicios y supermercados es superior a la del conjunto nacional; y el menor número de habitantes por establecimiento, en las tres clases, especialmente en cuanto a supermercados se refiere, denota una mejor dotación de ellos.

El detalle de estos establecimientos, por Agrupaciones, es como sigue:

\begin{tabular}{|c|c|c|c|}
\hline Agrupaciones & $\begin{array}{c}\text { Autoser- } \\
\text { vicios }\end{array}$ & $\begin{array}{c}\text { Superser- } \\
\text { vicios. }\end{array}$ & $\begin{array}{c}\text { Super- } \\
\text { mercados }\end{array}$ \\
\hline 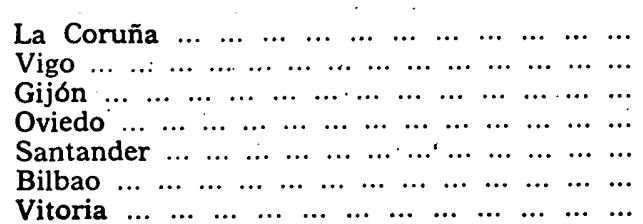 & $\begin{array}{r}75 \\
23 \\
90 \\
47 \\
70 \\
309 \\
25\end{array}$ & $\begin{array}{r}17 \\
20 \\
14 \\
9 \\
19 \\
58 \\
2\end{array}$ & $\begin{array}{l}3 \\
3 \\
2 \\
2 \\
4 \\
9 \\
2\end{array}$ \\
\hline
\end{tabular}




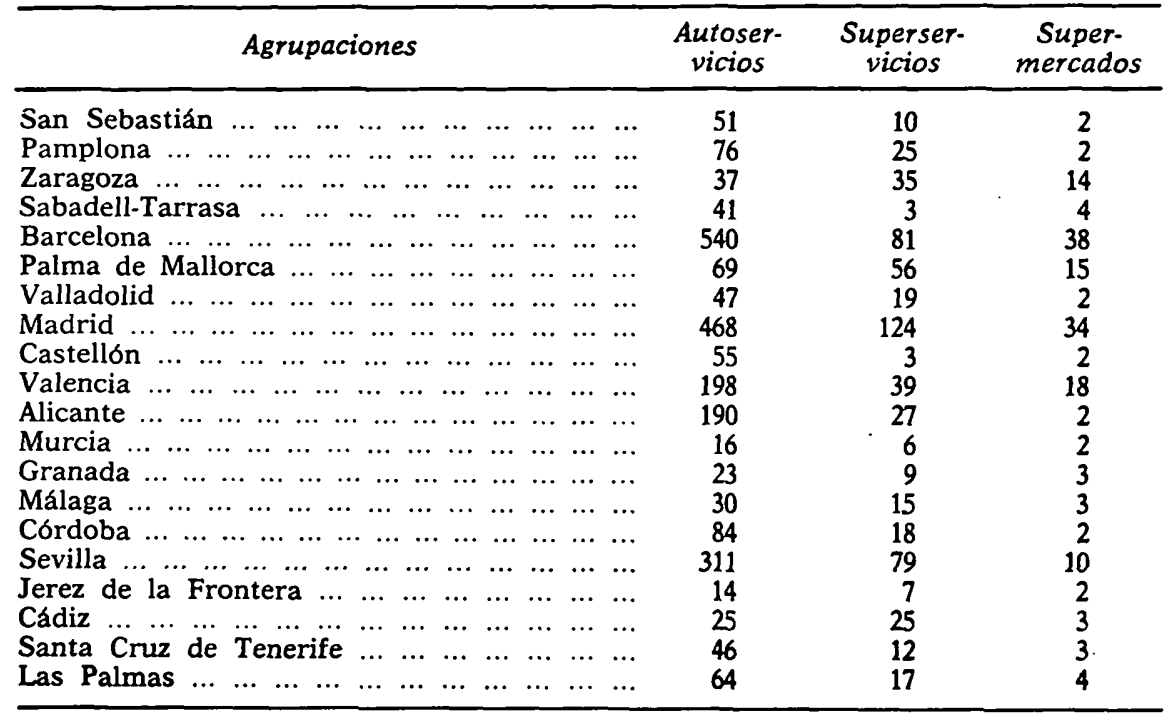

El estudio que comentamos contiene una amplia información para cada Agrupación, relativa a estos establecimientos por clases, superficie de venta, instalaciones frigoríficas, número de cajas, habitantes por establecimiento y por metro cuadrado y superficie media por establecimiento.

\section{ESTABLECIMIENTOS DE GRAN SUPERFICIE}

La distribución de estos establecimientos en el conjunto nacional y en las Agrupaciones es ésta:

\begin{tabular}{|c|c|c|c|}
\hline$\bullet$ & $\begin{array}{l}\text { Almacenes } \\
\text { populares }\end{array}$ & $\begin{array}{l}\text { Hiper- } \\
\text { mercados }\end{array}$ & $\begin{array}{l}\text { Grandes } \\
\text { almacenes }\end{array}$ \\
\hline Conjunto nacional: & & & \\
\hline 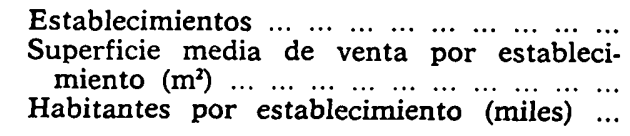 & $\begin{array}{c}122 \\
1.815,2 \\
294\end{array}$ & $\begin{array}{l}8.481,8 \\
3.258\end{array}$ & $\begin{array}{c}12.525,9 \\
703\end{array}$ \\
\hline Agrupaciones: & & ; & $\because$ \\
\hline $\begin{array}{l}\text { Establecimientos } \ldots \ldots \ldots \ldots \\
\text { Superficie media de venta por estableci- } \\
\text { miento }\left(\mathrm{m}^{2}\right) \ldots \ldots \ldots \ldots \ldots \ldots \\
\text { Habitantes por establecimiento (miles) } \ldots\end{array}$ & $\begin{array}{c}86 \\
1.862,0 \\
169\end{array}$ & $\begin{array}{l}9 \\
8.800,0 \\
1.618\end{array}$ & $\begin{array}{l}47 \\
13.262,7 \\
310\end{array}$ \\
\hline
\end{tabular}


Las Agrupaciones disponen del 70,5 por 100 de los almacenes populares, del 81,8 por 100 de los hipermercados y del 92,2 por 100 de los grandes almacenes. La superficie media de los establecimientos de todas clases en las Agrupaciones es superior a la del conjunto nacional, y el número de habitantes por establecimiento es notoriamente inferior, lo que indica que las Agrupaciones se hallan en condiciones óptimas en orden a la disponibilidad de estos establecimientos.

Los almacenes populares están muy generalizados; 24 Agrupaciones disponen de ellos; tan sólo Palma de Mallorca, San Sebastián y Santa Cruz de Tenerife carecen. La mitad se hallan en Madrid y Valencia. Madrid dispone de $33-38$ por 100 del total-, con una superficie media de venta de $1.597 \mathrm{~m}^{2}$ y un promedio de 113.000 habitantes por establecimiento; y Valencia tiene 12, con $1.766 \mathrm{~m}^{2}$ de superficie media de venta y 78.000 habitantes por establecimiento. Sevilla dispone de cinco, Barcelona de cuatro, Bilbao, Gijón y Murcia de tres cada una. Cádiz, Córdoba, Las Palmas, Sabadell-Tarrasa, Santander y Zaragoza tienen dos cada una, y las restantes Agrupaciones sólo tienen uno.

Los hipermercados se hallan situados: tres en Barcelona, con una superficie media de $9.400 \mathrm{~m}^{2}$ y 896.000 habitantes por establecimiento; dos en Madrid, con $7.500 \mathrm{~m}^{2}$ de superficie media y 1.863.000 habitantes por establecimiento. Málaga, San Sebastián, Sevilla y Valencia disponen de los restantes.

Tienen grandes almacenes 19 Agrupaciones. Madrid dispone de 15 , con una superficie media de venta de $12.241 \mathrm{~m}^{2}$ y 248.000 habitantes por establecimiento. Barcelona tiene cinco, con una superficie media de $19.565 \mathrm{~m}^{2}$ y 538.000 habitantes por establecimiento. Disponen de dos grandes almacenes: Bilbao, Córdoba, La Coruña, Murcia, Oviedo, Palma de Mallorca, Sevilla, Vitoria y Zaragoza; y de uno: Granada, Málaga, Las Palmas, Santander, Valladolid y Vigo.

\section{ESTIMACION DEL CONSUMO PRIVADO}

En el trabajo que comentamos se ha estimado el valor del consumo privado efectuado en las Agrupaciones, por las familias y las instituciones sin fines de lucro, por bienes y servicios en 1974 , en función de las cifras de la Contabilidad Nacional, la respectiva cuo- 
ta de mercado y los nuevos tipos de bienes y servicios considerados en la «Encuesta de Presupuestos Familiares 1973-74», del Instituto Nacional de Estadística, para núcleos de más de 50.000 habitantes.

Limitándonos al importe del consumo privado, total y por habitante, así concebido, sin analizar el detalle por bienes y servicios, los resultados globales son:

\begin{tabular}{|c|c|c|}
\hline & $\begin{array}{l}\text { Conjunto } \\
\text { nacional }\end{array}$ & Agrupaciones \\
\hline Consumo privado total (millones de ptas.). & $3.359 .921,1$ & $1.755 .346,8$ \\
\hline Consumo por habitante y año (ptas.) ... ... & 93.766 & 120.570 \\
\hline
\end{tabular}

advirtiéndose que el consumo efectuado en las Agrupaciones consideradas supone el 52,5 por 100 del total nacional, y que el consumo por habitante y año en las Agrupaciones resulta superior al nacional en un 28,6 por 100 .

El detalle por Agrupaciones es como sigue:

\begin{tabular}{|c|c|c|}
\hline \multirow{2}{*}{ Agrupaciones } & \multicolumn{2}{|c|}{ Consumo privado } \\
\hline & $\begin{array}{l}\text { Total millones } \\
\text { de pesetas }\end{array}$ & $\begin{array}{l}\text { Pesetas por } \\
\text { habitante } y \text { año }\end{array}$ \\
\hline 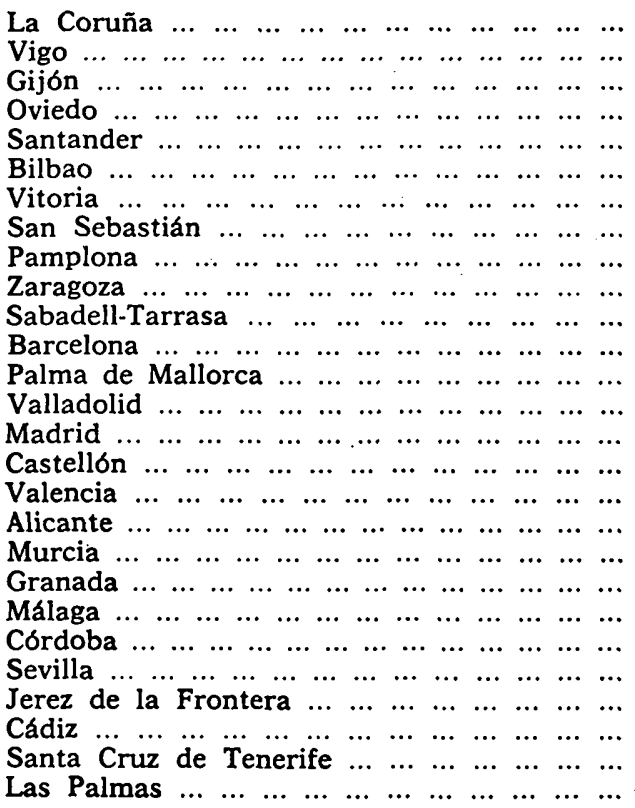 & $\begin{array}{r}25.837,8 \\
23.183,5 \\
24.863,4 \\
23.519,2 \\
23.385,1 \\
103.012,3 \\
18.239,8 \\
33.532,2 \\
22.209,1 \\
65.351,6 \\
31.820,0 \\
365.289,6 \\
43.640,0 \\
28.525,4 \\
485.542,3 \\
15.119,6 \\
115.346,1 \\
29.130,5 \\
24.258,6 \\
24.258,6 \\
44.989,0 \\
21.940 .3 \\
73.784,0 \\
11.087,8 \\
18.210,8 \\
24.090,7 \\
35.178,4\end{array}$ & $\begin{array}{r}105.004 \\
105.791 \\
119.500 \\
128.319 \\
113.923 \\
117.774 \\
115.301 \\
140.090 \\
117.599 \\
124.083 \\
86.182 \\
135.878 \\
167.734 \\
103.228 \\
130.153 \\
98.526 \\
123.399 \\
125.336 \\
83.836 \\
101.115 \\
108.881 \\
88.365 \\
98.328 \\
69.440 \\
88.313 \\
93.466 \\
115.036\end{array}$ \\
\hline
\end{tabular}


622 REVISTA DE ESTUDIOS DE LA VIDA LOCAI.

En líneas generales, el valor del consumo en cada Agrupación está en razón directa al número de sus habitantes. Mayor interés tiene contemplar el consumo por habitante y año, que en la mayor parte de las Agrupaciones, salvo seis, es superior al del conjunto nacional, en 19 de ellas supera las 100.000 pesetas y solamente en ocho rebasa al del conjunto de las Agrupaciones, figurando en primer lugar Palma de Mallorca, seguida de San Sebastián, Barcelona, Madrid, Oviedo, Alicante, Zaragoza y Valencia, enumeradas en orden descendente de valores.

La investigación sobre Agrupaciones urbanas contiene una más amplia información, en cuyo análisis pueden profundizar los estudiosos para obtener un conocimiento más cabal del tema. 
REVL-1977, núm. 195. BALLESTER ROS, IGNACIO. LAS AGRUPACIONES URBANAS ESPAÑOLAS ...

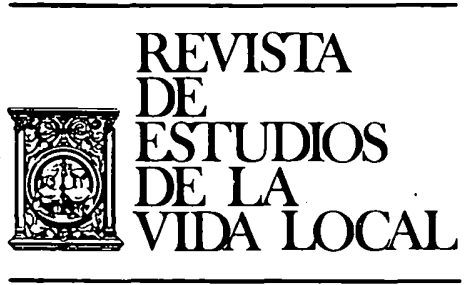

\section{JURISPRUDENCIA}


REVL-1977, núm. 195. BALLESTER ROS, IGNACIO. LAS AGRUPACIONES URBANAS ESPAÑOLAS ... REVL-1977, núm. 195. BALLESTER ROS, IGNACIO. LAS AGRUPACIONES URBANAS ESPAÑOLAS ... 